\title{
IR-RADIO SPECTRAL INDICES FOR Be STARS
}

\author{
H. CHEN and A. R. TAYLOR \\ University of Calgary, Canada \\ S.M. DOUGHERTY \\ John Moores University of Liverpool, UK \\ and \\ L.B.F.M. WATERS \\ SRON Laboratory for Space Research, The Netherlands
}

\begin{abstract}
Using published data and new data from $\mathrm{mm}$ observations, we calculate spectral indices $\alpha(12 \mu-25 \mu), \alpha(25 \mu-1.1 \mathrm{~mm})$ and $\alpha(1.1 \mathrm{~mm}-2 \mathrm{~cm})$ for Be stars. The index $\alpha(25 \mu-$ $1.1 \mathrm{~mm}$ ), obtained for 8 stars, shows two characteristics: 1) for "normal" Be stars the index decreases from earlier types towards later types, i.e., later type Be stars tend to have shallower spectra in the IR-radio region than earlier types; 2) the index for shell stars appears to have smaller values than that for normal Be stars with same spectral types.
\end{abstract}

\section{Introduction}

In an initial VLA survey carried out by Taylor et al. (1987), five Be stars were observed at $\lambda=6 \mathrm{~cm}$, resulting in the detection of one star, $\psi$ Per, the only shell star in the program stars. In a second radio survey (Taylor et al. 1990, Taylor survey, hereafter), 21 Be stars were observed at $\lambda=2 \mathrm{~cm}$, among which five are shell stars and sixteen others are "normal" Be stars. Six stars were detected. The detection rate at radio is $60 \%$ and $19 \%$ for shell stars and "normal" Be stars, respectively. This may suggest that shell stars are more likely to be radio emitters than normal Be stars.

In Taylor survey, the distribution of program stars is similar to the frequency of Be star, i.e., it peaks at B2 and decreases towards both directions. However the detected stars do not show any preference to B2. In fact, the detection rate for the stars later than $\mathrm{B} 5$ is $3 / 7=43 \%$ relative to $3 / 14=21 \%$ for the stars earlier than B5. This suggest that stars of later types might have relatively stronger emission at radio wavelengths than stars of earlier types.

In this investigation, we re-examine IR, $\mathrm{mm}$ and radio spectral indices for Be stars to see if there is any difference between "normal" Be stars and shell stars and between Be stars of earlier and later spectral types.

\section{Results}

The infrared spectral indices, $\alpha(12 \mu-25 \mu)$, for 58 stars show no dependence on spectral types, although the values for earlier types tend to be more scattered than those for later types. Neither does it show dependence on 


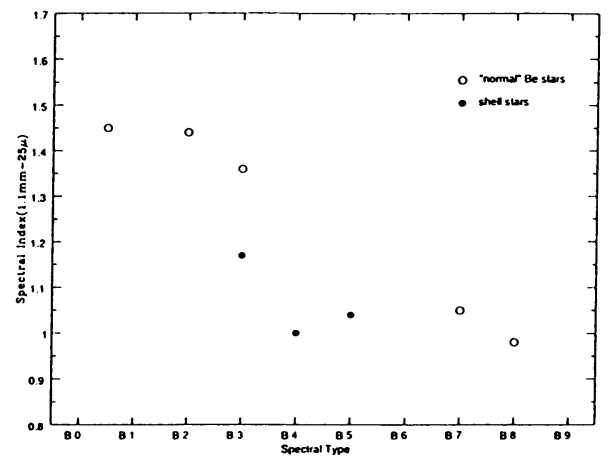

Fig. 1. Spectral index $\alpha(25 \mu-1.1 \mathrm{~mm})$ versus spectral types for eight stars.

whether the star is a shell star or a normal Be star. This is consistent with the fact that the distribution of progam stars in Taylor survey is similar to the relative frequency of Be stars, since the program stars in Taylor survey were chosen according to their flux in the IRAS data.

In Fig. 1, we plot the infrared-millimeter spectral index, $\alpha(25 \mu-1.1 \mathrm{~mm})$, for eight stars. The index shows two characteristics: 1) for "normal" Be stars the index decreases towards later types, i.e., later type Be stars tend to have shallower spectra in the IR-radio region than earlier types; 2 ) the index for shell stars appears to have smaller values than that for normal Be stars with same spectral types. The first characteristic suggests that the density in the envelopes of later type Be stars may drop less fast with the increasing distance from the underlying star than that for earlier types. Therefore, later type stars tend to emit more towards longer wavelengths relative to earlier type stars. This would explain why the detection ratio appears higher for later type stars in the Taylor survey. The second characteristic suggests that shell stars have more emitting material at larger distance from the underlying star than "normal" Be stars. As the result, one would expect that shell stars are more likely to be detected at radio, which is consistent with the result in Taylor survey.

This result is supported by the cm-mm spectral index $\alpha(1.1 \mathrm{~mm}-2 \mathrm{~cm})$ calculated for six stars. For "normal" Be stars, the index tends to have about the same value of 1.4 , while the index for shell stars tends to be smaller and more scattered.

\section{The References}

Taylor, A.R., Waters, L.B.F.M., Lamers, H.G.L.M., Persi, P., \& Bjorkman, K.S., Mon. Not. R. ast. Soc. 288, 811 (1987).

Taylor, A.R., Waters, L.B.F.M., Bjorkman, K.S. \& Dougherty, S.M., Astr. Astrophys. 231, 453 (1990). 172, 225 (1987). 